\title{
Eine Bedrohung für den Sinn? Human Enhancement und das sinnvolle Leben
}

\author{
Markus Rüther
}

Eingegangen: 12. April 2021 / Angenommen: 23. Juli 2021 / Online publiziert: 20. September 2021

(C) Der/die Autor(en) 2021

Zusammenfassung Das allgemeine Thema des Artikels besteht darin, zwei Diskussionskreise miteinander zu verbinden, die in Isolation betrachtet gut erforscht sind, aber selten zusammengebracht werden: die Diskussion um das sogenannte Human Enhancement und die Debatte um das sinnvolle Leben. Hierbei wird insbesondere die Behauptung ins Blickfeld gerückt, dass Techniken des Human Enhancement einen negativen Einfluss auf den Sinn haben könnten. Ist diese These plausibel? In diesem Artikel wird die These verteidigt, dass diese Frage verneint werden muss. Methodisch werden hierfür drei Varianten untersucht, in denen die Behauptung des negativen Einflusses vorgetragen werden können. Dabei wird aufgezeigt, dass entweder die Annahme eines negativen Einflusses aus empirischen Gründen wenig wahrscheinlich ist oder dass sie durch Modifikationen der Enhancement Techniken abgeschwächt werden können.

Schlüsselwörter Lebenssinn · Enhancement · Wohlergehen

Dr. phil. Markus Rüther $(\square)$

Institut für Neurowissenschaften und Medizin, Ethik in den Neurowissenschaften (INM-8),

Forschungszentrum Jülich GmbH, 52425 Jülich, Deutschland

E-Mail: m.ruether@fz-juelich.de 


\title{
A threat for meaningfulness? Human enhancement and the meaningful life
}

\begin{abstract}
Definition of the problem The general theme of this article is the connection between two topics which are well-researched on their own, but are hardly linked to each other: the debate on human enhancement and the meaning in life debate. More precisely, the article explores the claim whether there is reason to believe that enhancement techniques can undermine the meaning of one's life.

Arguments How credible is this thesis? In this article, it is argued: "not very". This thesis is elaborated by evaluating three different versions of the negative impact thesis.

Conclusion In each case it is argued that the negative impact claim either fails outright or, at best, provides weak and defeasible grounds for avoiding enhancement.
\end{abstract}

Keywords Meaning in life $\cdot$ Enhancement $\cdot$ Well-being

\section{Vorbemerkungen}

Kann das Human-Enhancement dem Leben seinen Sinn rauben? Tatsächlich haben einige Autoren dafür argumentiert, dass diese Frage bejaht werden muss. Sie meinen, dass alle Techniken, die wir mit dem Enhancement verbinden, mindestens eine Sache gemeinsamen haben: Sie alle haben eine sinnminimierende Kraft, die derart stark ist, dass die Sinnbilanz eines Lebens ernsthaft leidet oder - im Extremfall - sogar negativ ausfallen kann. Diese Kritiklinie bildet den Hauptgegenstand dieses Beitrages. Um sie zu evaluieren, werde ich in drei Schritten vorgehen:

In einem ersten Schritt werde ich den Problemkontext der Kritik noch genauer erläutern, mein Verständnis der Begriffe „Human Enhancement“ und „,sinnvolles Leben" darlegen, insbesondere aber auch einige Einschränkungen der noch sehr allgemein gehaltenen Fragestellung vornehmen. In einem zweiten Schritt werden sodann drei vermeintliche ,Sinnräuber“ in den Blick genommen, die in der Literatur diskutiert werden. Hierbei werden zwei Schlussfolgerungen herausgearbeitet: Zum einen wird sich zeigen, dass es nicht sonderlich wahrscheinlich ist, dass die sinnminimierende Kraft der Enhancement-Technologien tatsächlich auftritt. Zum anderen kann der Sinnverlust, insofern er sich dennoch abzeichnet, mit geeigneten Gegenmaßen eingedämmt und gemildert werden. In einem dritten Schritt werden diese Ergebnisse weiter präzisiert, indem einige kritische Nachfragen, aber auch einige noch offene Anschlussfragen erläutert und diskutiert werden.

\section{Problemkontext, Terminologie und Fragestellung}

Den Ausgangspunkt dieses Beitrages bildet die Vermutung, dass es eine interessante thematische Verbindung zwischen zwei Diskursen gibt, die nur selten zusammen behandelt werden - die Debatte um das sogenannte Human Enhancement einerseits 
und um das sinnvolle Leben andererseits. ${ }^{1}$ Um einige terminologische Klärungen vorauszuschicken: Der Begriff „Enhancement“ ist semantisch vieldeutig. Ich verstehe darunter im Folgenden eine bewusste Anwendung von technischem und wissenschaftlichem Wissen, um menschliche Fähigkeiten in einer Weise zu verbessern oder neue zu schaffen, sodass diese über der Leistungsfähigkeit von normalen Fähigkeiten liegen, die jemand ohne die Anwendung eines solchen Wissens hätte. ${ }^{2}$ Darüber hinaus möchte ich mit den Begriffen „Enhancement“ oder - wie man das Phänomen manchmal auch umschreibt - „Selbstverbesserung“ und „Selbstoptimierung“ nicht bereits aus begriffslogischen Gründen eine positive Bewertung verbinden. $\mathrm{Ob}$ es sich tatsächlich um eine positive oder negative Veränderung handelt, soll durch substanzielle Argumente und nicht durch die Semantik entschieden werden. Unter dem ,Enhancement Projekt“ ${ }^{3}$ verstehe ich den Titel für eine Unternehmung, die sich nicht auf einen einzigen Enhancement-Eingriff bezieht, sondern auf eine Reihe von Versuchen, die dem Ziel gewidmet sind, sich selbst zu verbessern. Entsprechend werden auch solche Eingriffe ausgeschlossen, die auf andere ausgerichtet sind (z. B. auf den eigenen Nachwuchs). Weiterhin möchte ich auch nur solche Maßnahmen darunter fassen, bei denen es sich um biomedizinische Eingriffe handelt. Das umfasst Praktiken wie Schönheitsoperationen, smart drugs, Brain-Maschine-Interfaces und die Implantation von bionischen Gliedmaßen oder KI-Chips. ${ }^{4}$

Damit zum Begriff des ,sinnvollen Lebens“: Hierbei handelt sich um ein begriffliches Syntagma, das ich aus einem Diskurs übernehme, welcher in der analytischen Philosophie unter dem Titel „Meaning-in-Life“ bekannt geworden ist. ${ }^{5}$ Was verstehen die Hauptprotagonisten dieses Diskurses darunter? Das kann recht unterschiedlich sein. Als kleinster gemeinsamer Nenner kann jedoch die Ansicht fungieren, dass das sinnvolle Leben eine eigenständige Wertdimension des individuell guten Lebens darstellt. Das muss nicht heißen, dass der Sinn gar nicht durch andere Wertdimensionen, zum Beispiel durch das Wohlergehen oder die Moral, beeinflusst wird. Wichtig ist jedoch, dass er nicht vollständig durch diese anderen Dimensionen erfasst wird. Es bleibt mithin immer ein gewisser phänomenologischer Rest. ${ }^{6}$ Als Beispiele solcher Sinntätigkeiten, die eine gewisse Eigenständigkeit haben, werden von Vertreterinnen etwa Tätigkeiten genannt wie das Schaffen von Kunstwerken, das Aufstellen einer bedeutsamen wissenschaftlichen Theorie oder die Hilfeleistung

\footnotetext{
1 Eine Ausnahme von der Regel bildet m. W. lediglich Kipke (2011, Kap. 6.4.3) und Danaher (2014), die beide diese Verbindung ebenfalls explizit herstellen.

2 Vgl. für eine ähnliche Definition auch Buchanan (2011, S. 23).

3 Vgl. für den Begriff des „Enhancement-Projekts“ den Titel des Buches von Hauskeller (2013). In einer ähnlichen Weise spricht Buchanan von einem „Enhancement-Enterprise“, wenn es um einen kollektiven Sammelbegriff geht. Vgl. Buchanan (2011).

4 Vgl. für die verschiedenen technischen Möglichkeiten des Enhancement etwa den Sachstandsbericht Heinrichs et al. (2021, Kap. 1).

5 Vgl. für eine genauere problemgeschichtliche Einordnung und Definition des Syntagmas die Ausführungen im Editorial dieses Themenhefts.

6 Hierbei bleibt offen, ob Wohlergehen, Moral und Sinn eine erschöpfende Trias für die Bestimmung des guten Lebens bilden. Behauptet wird von den meisten Vertretern lediglich die schwächere These, dass - um einen Untertitel eines Aufsatzes von Wolf zu paraphrasieren (A Third Dimension of the Good Life) die Sinndimension eine dritte, also zusätzliche Dimension des guten Lebens ist. Vgl. Wolf (2016b).
} 
gegenüber Bedürftigen. ${ }^{7}$ Die Forschungsfragen, die an solche Sinntätigkeiten herangetragen werden, sind vielfältig. Dazu gehören metaethische Fragen nach den sprachphilosophischen und ontologischen Grundlagen des Sinnbegriffs, aber auch normativ-ethische, welche die Frage nach den konkreten Sinnquellen oder ",Sinnräubern" des sinnvollen Lebens betreffen. ${ }^{8}$ In jedem Fall handelt es sich beim Meaningin-Life-Diskurs um ein lebhaftes Forschungsfeld, welches - wenn es nicht bereits als etablierter Bestandteil gelten kann - in der Philosophie immer mehr an Aufmerksamkeit gewinnt.

Wie aber können diese beiden Diskurse zusammengebracht werden? Es gibt verschiedene Möglichkeiten. ${ }^{9}$ Eine interessante Frage, der ich im Folgenden weiter nachgehen möchte, betrifft den normativen Einfluss, den Enhancement-Eingriffe auf die Sinnbilanz eines Lebens haben könnten. Oder noch etwas genauer: Mich interessiert vor allem der negative Einfluss. Lassen sich im Enhancement-Projekt möglicherweise einige Faktoren ausmachen, welche die Sinnbilanz eines Lebens erheblich herabstufen können? Um an dieser Stelle eine Einschränkung vorzunehmen: Im Rahmen einer solchen Fragestellung kann ich nicht alle potenziellen „Sinnräuber“ in den Blick nehmen. Ich werde mich daher auf eine einzige, aber sehr traditionelle Klasse von Gesichtspunkten beschränken, die häufig unter dem Begriff „Wohlergehen " zusammengefasst werden. ${ }^{10}$ Als Sammelbegriff bezieht sich das Wohlergehen, wie der Name schon sagt, auf das menschliche Wohl. Es beinhaltet all diejenigen Dinge, die nicht für andere, sondern nur für das Individuum gut sind. Inhaltlich kann die Bestimmung heterogen ausfallen. Von Derek Parfit stammt etwa eine klassische, aber immer noch häufig verwendete Unterteilung in hedonistische Theorien, Wunschtheorien und Objektive-Listen-Theorien. ${ }^{11}$ Um die Kernthesen anzudeuten: Eine hedonistische Theorie behauptet im Wesentlichen, dass das Wohlergehen durch bestimmte, positive mentale Zustände beschrieben werden kann. Eine Wunschtheorie vertritt die These, dass unser Wohlergehen durch Wünsche und deren Erfüllung gekennzeichnet ist. Und eine Objektive-Listen-Theorie meint, dass es bestimmte von subjektiven Einstellungen unabhängige Gegenstände unseres Handelns sind, die das menschliche Wohl charakterisieren. Nun ist mir nicht so wichtig, ob Parfit damit eine hinreichende Systematik liefert. Wichtiger ist, dass dadurch der Begriff des Wohlergehens für die weiteren Zwecke des Beitrages verständlicher wird und eine

\footnotetext{
${ }^{7}$ Manche Autoren sprechen daher zusammenfassend von der Trias des Schönen, Wahren und Guten, die den Kernbereich des sinnvollen Lebens ausmachen soll. Vgl. für eine Verwendung der Trias als Ausgangspunkt für die Ausarbeitung einer eigenen Sinntheorie Metz (2013a).

8 Vgl. dafür etwa die bereits klassischen Literaturberichte in Metz (2002, 2007, 2013b). Für eine Bestandsaufnahme des neueren Diskurses siehe Rüther (2021a, b).

9 Vgl. dafür etwa den Überblicksartikel von Juengst und Moseley (2021) im Oxford Companion zum Thema „Meaning-in-Life“.

10 „Sehr traditionell“ meint in diesem Zusammenhang vor allem, dass nahezu alle Theoretiker davon ausgehen, dass zumindest einige Aspekte des Wohlergehens alleine oder im Verbund mit anderen Aspekten notwendige Bedingungen des sinnvollen Lebens sind. Vgl. für diese Analyse etwa den Literaturbericht Rüther (2021b). Eine Ausnahme von der Regel bilden Smuts (2013) und Rüther M (i. V.), die das sinnvolle Leben vom Wohlergehen zu entkoppeln versuchen, da beide dafür argumentieren, dass ein sinnvolles Leben ohne irgendeinen Aspekt des Wohlergehens möglich ist.

11 Vgl. Parfit (1984, S. 493). Vgl. für die Verwendung der Dreiteilung etwa Haybron (2008, S. 34), Keller (2009, S. 84), Rice (2013, S. 199), Fletcher (2015, S. 3) und Moore (2015).
} 
schärfere Kontur bekommt. Wenn ich im Weiteren davon spreche, dass es bestimmte sinnminimierende Aspekte gibt, dann meine ich damit ausschließlich solche, die das Wohlergehen betreffen, also für das Individuum gut sind; das können zum Beispiel - wie Parfit meint - seine hedonischen Zustände, seine erfüllten Wünsche oder ein realisiertes objektives Gut sein; es können aber auch, wenn man eine andere Theorie zugrunde legt, noch zusätzliche oder ganz andere Dinge sein. Diejenigen sinnminimierenden Faktoren, die im Folgenden eine Rolle spielen, werden sich - wie noch veranschaulicht wird - indes gut in das Parfit'sche Schema einordnen lassen.

Es ist wichtig zu beachten, dass diese und weitere Aspekte des Wohlergehens nicht irgendeine mögliche Facette des sinnvollen Lebens darstellen. Nahezu alle Autorinnen und Autoren in der Meaning-in-Life-Debatte gehen davon aus, dass ein oder mehrere Aspekte des Wohlergehens notwendige Bedingungen für Sinn darstellen. ${ }^{12}$ Das muss nicht bedeuten, dass nicht noch weitere Aspekte hinzutreten müssen, die nichts mit dem Wohlergehen zu tun haben, um ein Leben in vollwertiger Weise sinnvoll zu machen. Ebenso wenig ist damit die These verbunden, dass es sich beim Wohlergehen um das wichtigste Kernelement handelt. Es wird lediglich behauptet: Wenn wir ein sinnvolles Leben führen wollen, dann muss ein Aspekt des Wohlergehens darin auch eine Rolle spielen, weil sonst die Minimalbedingungen für Sinn nicht erfüllt sind.

Um zum Abschluss noch zwei methodische Einschränkungen diese Beitrages zu erwähnen: Zum einen werde ich ausschließlich die sinnminimierende Kraft von Gesichtspunkten des Wohlergehens untersuchen, die dem Anspruch nach einen direkten Einfluss auf den Sinn haben. Solche, die das erst über einen Umweg tun (z. B. über gesellschaftliche oder soziale Bedingungen) werden außer Acht gelassen. Zum anderen werde ich nicht infrage stellen, ob die zu prüfenden Aspekte tatsächlich die sinnminimierende Kraft besitzen, wie die jeweiligen Autoren behaupten. Das würde in eine Diskussion darüber führen, welche Inhalte für das sinnvolle Leben überhaupt relevant sind und welche nicht. Diese Debatte möchte ich an dieser Stelle aussparen..$^{13}$ Stattdessen möchte ich zugunsten der Kritiker des Enhancement-Projekts voraussetzen, dass die jeweiligen Gesichtspunkte tatsächlich sinnminimierend wirken können, und prüfen, ob es plausibel erscheint, diese mit dem EnhancementProjekt in Verbindung zu bringen. Mit anderen Worten: Es geht mir nicht um die Frage, ob einige Aspekte des Wohlergehens tatsächlich potenzielle "Sinnräuber“ sind, sondern - wenn wir das einmal zugestehen - um die Frage, ob das für das Enhancement-Projekt überhaupt ein Problem darstellt.

\footnotetext{
12 Vgl. den Literaturbericht in Rüther und Muders (2016), der die Relevanz des Wohlergehens für den Sinn hervorhebt, wenngleich die konkrete Interpretation der Valenz im Vergleich zu anderen Aspekten erheblich voneinander abweichen kann.

${ }^{13}$ Meine eigene Sinntheorie findet sich in Rüther M (i. V.).
} 


\section{Die ,Sinnräuber“6}

\section{Dauerhafte Unzufriedenheit}

Ein erster Versuch, das Enhancement-Projekt als sinnminimierend zu entlarven, stammt von Michael Hauskeller. Den Ausgangspunkt seiner Überlegungen bildet die Rekonstruktion und Modifikation des von Michael Sandel entwickelten „Giftedness of Life"-Arguments. ${ }^{14}$ Sandel drückt darin seine Bedenken aus, dass das Enhancement-Projekt die Wertschätzung für die geschenkhaften Aspekte unseres Lebens unterminieren könnte. ${ }^{15}$ Ein solches „Geschenk“ seien etwa unsere Talente und Fähigkeiten sowie alle weiteren Dinge, die uns von der Natur mitgegeben wurden und für uns gut sind. Warum ist die mangelnde Wertschätzung ein Problem? Das ist nicht so leicht zu beantworten und Sandel scheint in diesem Zusammenhang nicht immer ganz eindeutig und unmissverständlich zu argumentieren. ${ }^{16}$ Hauskeller hält die grobe Argumentationslinie jedoch weiterhin für tragfähig und gibt verschiedene Hinweise, worin das Problem bestehen könnte. ${ }^{17}$ Für die Zwecke dieses Beitrages sind nur diejenigen Antworten von Bedeutung, die sich auf die Aspekte des Wohlergehens zurückführen lassen, wenngleich sich auch andere nicht-wohlergehenszentrierte Aspekte ausmachen ließen. ${ }^{18}$ Eine solche sind Hauskellers Bedenken, dass das Enhancement-Projekt zu einem dauerhaften Zustand der Unzufriedenheit mit sich selbst und einem Leiden an der eigenen Unvollkommenheit führt und daher an eine im weitesten Sinne hedonistische Theorie des Wohlergehens anknüpft. ${ }^{19}$

Wie lässt sich dieser Zusammenhang herstellen? Das kann man erkennen, wenn man Hauskellers zentrale Ausgangsprämisse in den Blick nimmt. Diese besteht darin, dass er das Enhancement-Projekt durch den abgewandelten Voltair'schen Grundsatz Das Bessere ist der Feind des Guten (Original: Le mieux est l'ennemi du bien) gekennzeichnet sieht. ${ }^{20}$ Dieser Grundsatz sei mit einer relativistischen These bezüglich intrinsischer Werte verbunden, die es unmöglich mache, noch an der Vorstellung festzuhalten, dass etwas an sich gut sei. Oder in einfachen Worten ausgedrückt: Die bloße Möglichkeit, dass am Horizont des technologischen Fortschritts immer noch eine erfolgversprechendere Selbstverbesserung warten könnte, führt dazu, dass wir dasjenige, was wir bereits haben, nicht mehr wertschätzen können. ${ }^{21}$ Das Gute, das

\footnotetext{
14 Vgl. Sandel (2007).

15 Sandel (2007, S. 26f.).

16 Das sieht etwa auch Hauskeller so und hält unmissverständlich mit Blick auf die Präsentation und Logik des Arguments von Sandel fest: „Frankly, this whole train of reasoning does not sound like a conclusive argument at all“ (Hauskeller 2011, S. 58).

17 Vgl. Hauskeller (2011) und Hauskeller (2013, Kap. 10).

18 Ein Beispiel dafür bildet etwa die von Hauskeller verwendete Floskel des „,being in the world“, welche dazu einlädt, auch sinnminimierende Faktoren zu berücksichtigen, die prima facie wenig mit dem eigenen Wohl zu tun haben. Für den diesen Hinweis bedanke ich mich bei einem anonymen Gutachter.

19 Vgl. für eine differenziertere Analyse von hedonistischen Wohlergehenstheorien auch Rüther und Muders (2016).

20 Hauskeller (2011, S. 71).

21 Er schreibt explizit: ,(E)verything that excites, fascinates and enraptures us today will no longer have any interest to us whatsoever" (Hauskeller 2011, S. 72).
} 
wir haben, ist niemals mehr gut genug. Das Bessere wird, wie der Slogan besagt, zum Feind des Guten. Und diese Einstellung führt, so lässt sich Hauskeller interpretieren, zu einer dauerhaften Unzufriedenheit mit uns selbst (z.B. mit den eigenen unvollkommenen Talenten und Fähigkeiten), aber auch mit den unvollkommenen Dingen, die es in der Welt gibt (z. B. mit Kunstwerken).

Was ist von diesem Argument zu halten? Sehen wir einmal von einigen terminologischen und logischen Herausforderungen ab, möchte ich drei allgemeine Dinge anmerken $^{22}$ : Erstens scheint es mir nicht ausgemacht zu sein, dass sich der von Hauskeller diagnostizierte Relativismus auf alle Werte beziehen muss. Es scheint vielmehr so, dass zumindest der intrinsische Wert der Selbstverbesserung nicht tangiert wird, da dieser dem Enhancement-Projekt zugrunde liegt. Nun mag das für Hauskeller nicht genug sein, weil er andere Werte, die vom Enhancement-Projekt bedroht werden (z.B. den Wert der natürlich gewachsenen Talente und Anlagen) für Sinnquellen hält und damit deren Elimination für einen erheblichen sinnminimierenden Faktor. Das scheint aber mitnichten für alle Theoretiker selbstevident zu sein, sondern im Gegenteil sogar ein Grund, das Enhancement-Projekt fortzuführen. ${ }^{23} \mathrm{Im}$ Klartext: Ein einfacher Sieg ist für Hauskeller an dieser Front nicht zu erwarten, sondern verlangt nach weiteren substanziellen Argumenten.

Zweitens scheint mir Hauskeller zu übertreiben, wenn er davon ausgeht, dass das Enhancement-Projekt dazu führt, dass für jedes Individuum nahezu ${ }^{24}$ alle intrinsischen Werte auf dem Prüfstand stehen und relativiert zu werden drohen. Das mag vielleicht auf die kühnsten Techno-Utopisten zutreffen, die schlicht alles zum Gegenstand der Verbesserung machen wollen. Für den ,normalen“ EnhancementAnwender scheint die globale Selbstverbesserung, wie Frances Kamm feststellt, jedoch wenig attraktiv zu sein. ${ }^{25}$ Für diese Gruppe von Menschen kann man eher davon ausgehen, dass sie nur ausgewählte Bereiche optimieren bzw. verbessern wollen. Das bedeutet aber auch, dass diese nicht in einem globalen Sinne von der Vorstellung des it could be better infiziert werden, sodass eine dauerhafte Unzufriedenheit bei ihnen nicht in gleichem Maße anzutreffen sein dürfte. Kurzum: Es verbietet sich für Hauskeller, von einer verallgemeinerbaren These zu sprechen, wenn er die dauerhafte Unzufriedenheit ins Feld führt. Diese mag, wenn überhaupt, nur auf einen kleinen, besonders technikaffinen Personenkreis zutreffen. Ein allgemeiner Strick für alle Enhancement-Anwender lässt sich daraus aber nicht drehen.

Drittens wäre auch selbst für den technikaffinen Enhancer zu überlegen, wie schwer die von Hauskeller diagnostizierte Sinnminimierung tatsächlich wiegt. ${ }^{26}$ Klar ist jedenfalls, dass sich daraus kein Dammbruch-Argument ergibt. Nur, weil es eine

\footnotetext{
22 Vgl. für eine Kritik an einigen handwerklichen Details der Argumentation Danaher (2014, S. 237).

23 Eine solche Position erläutert Hauskeller auch selbst, ohne sich dann jedoch argumentativ mit ihr auseinanderzusetzen. Er greift lediglich ein Zitat des Transhumanisten Nick Bostrom heraus und erläutert davon ausgehend seine eigene Gegenposition, die - wenn sie als plausibel ausgewiesen werden könnte den Wert der eigenen Talente und Anlagen freilich ganz anders beurteilen würde. Vgl. Hauskeller (2011, S. 72).

24 „Nahezu“ wird an dieser Stelle verwendet, da man - wie oben schon erläutert - zumindest die Selbstverbesserung als intrinsischen Wert von der Relativismuskritik ausnehmen muss.

25 Vgl. Kamm (2009).

26 Auf diesen wichtigen Punkt hat mich ein anonymer Gutachter aufmerksam gemacht.
} 
dauerhafte Unzufriedenheit gibt, muss das nicht bedeuten, dass unser Leben sinnlos wird. Gut möglich, dass es noch weitere sinnfördernde Faktoren gibt, die diese aufwiegen können. Ebenso könnte es sein, dass sinnfördernde Elemente vielleicht sogar als Nebenprodukte auftreten und dadurch für eine insgesamt positive Sinnbilanz sorgen. Am Ende könnte die dauerhafte Unzufriedenheit also für den Sinn gar nicht so negativ sein, wie Hauskeller meint. Allerdings: Um das abschließend zu bewerten, ist eine umfassende Sinntheorie notwendig, die jenseits dieses Beitrages liegt. An dieser Stelle muss diese aber auch gar nicht vorausgesetzt werden. Es reicht vielmehr festzustellen, dass es - anders als Hauskeller meint - keineswegs auf der Hand liegt, dass die dauerhafte Unzufriedenheit ein ernstzunehmender ,Sinnräuber“ ist.

\section{Entscheidungskonfusion, residuales Bedauern und moralische Schuld}

Einen weiteren Versuch, diejenigen Faktoren zu identifizieren, die einen negativen Einfluss auf den Sinngehalt unseres Lebens haben könnten, liefert Saskia Nagel in ihrem Aufsatz Too Much of a Good Thing? Enhancement and the Burden of SelfDetermination (2010). Darin geht sie - anders als Hauskeller - nicht davon aus, dass das Enhancement-Projekt von einer dauerhaften Unzufriedenheit mit den eigenen Talenten gekennzeichnet ist, sondern von negativen Erfahrungen wie Entscheidungskonfusion, residualem Bedauern und moralischer Scham. Nagel nennt damit Aspekte des Wohlergehens, die sowohl einer hedonistischen als auch wunschbasierten Theorien zugeordnet werden können. Den inhaltlichen Ausgangspunkt bildet für sie die Feststellung, dass durch die neuen Enhancement-Techniken zusätzlicher Entscheidungsspielraum entstanden ist. Vieles, was früher noch unverfügbar war, kann zum Gegenstand der Verbesserung gemacht werden. Das mag auf den ersten Blick auch zu begrüßen sein. Auf den zweiten Blick ist das jedoch nicht mehr so eindeutig, denn ,studies from psychology and consumer research teach us that the increase in possibilities might turn out not to be for our best and in fact hinder human flourishing“ (Nagel 2010, S. 110). Wie ist der Zusammenhang herzustellen? Betrachten wir die verschiedenen mentalen Zustände der Reihe nach. Zunächst zur Konfusion: Dafür stützt sich Nagel auf die bekannten empirischen Studien von Barry Schwartz zum sogenannten paradox of choice. ${ }^{27}$ Schwartz hatte festgestellt, dass die liberale Ansicht, die Bürgerinnen und Bürger mit immer mehr Wahlmöglichkeiten auszustatten, nicht immer mit optimalen Folgen für ihr Wohlergehen und damit auch für die Sinnbilanz einhergeht. Vielmehr sei ab einem bestimmten Punkt eine erhöhte Anzahl an Entscheidungsmöglichkeiten suboptimal, da diese mit negativen Gefühlen wie Konfusion einhergingen und sogar zur Entscheidungsmüdigkeit oder -paralyse führten. Die Gleichung ist also relativ einfach: mehr Optionen, mehr Konfusion, weniger Sinn. Allerdings: Die Studien von Schwartz sind nicht unwidersprochen geblieben. In einer Metaanalyse von 50 Studien zu demselben Thema haben etwa Scheibehenne et al. (2010) herausgearbeitet, dass nicht alle Studien zu demselben Ergebnis kommen, also entweder keine oder gegenteilige Zusammenhänge ermittelt haben. Der Zusammenhang zwischen einer Steigerung von Wahlmöglichkeiten und

27 Schwartz (2004). 
verstärkter Konfusion scheint mithin nicht ganz so eindeutig zu sein. Aber selbst wenn wir die empirische Validität nicht infrage stellen, kann bezweifelt werden, dass es sich bei der Konfusion um ein unüberwindliches Hindernis handelt. Gut belegt ist etwa, dass einfache Entscheidungsheuristiken, aber auch eine mentale Arbeit am eigenen mindset oder zumindest eine vage Vorstellung vom Ausgang einer Wahlsituation den Effekt erheblich mildern können. ${ }^{28}$ Es ist mithin kein Naturgesetz, dass die zusätzlichen Entscheidungsoptionen zur Konfusion und damit zu Sinneinbußen führen müssen.

Damit komme ich zum zweiten Aspekt, dem residualen Bedauern: Die Zusammenhänge erscheinen zunächst wieder plausibel zu sein. Wer mehr Wahlmöglichkeiten hat, hat auch mehr Möglichkeiten, seine Entscheidungen hinterher zu bedauern. Aber ist das Bedauern von Fehlentscheidungen tatsächlich immer sinnminimierend? Das kann man mit guten Gründen bezweifeln. In vielen Fällen kann das Bedauern einer Entscheidung auch eine kraftvolle Motivation sein, in der Zukunft bessere Entscheidungen zu treffen. Aber gehen wir das Argument von Nagel einmal mit. Wenn wir das tun, lässt sich erkennen, dass der Zusammenhang ,,mehr Entscheidungen - mehr Bedauern - weniger Sinn“ nicht in Stein gemeißelt ist. Vielmehr kann das Enhancement-Projekt in einer Weise angepasst werden, dass es Mittel bereitstellt, um den Leidensdruck, der von residualem Bedauern ausgeht, in einer beträchtlichen Weise abzumildern. ${ }^{29}$ Eine Option hierfür wäre die Forschung an den Möglichkeiten des Einsatzes von memory dampening. Diese Technologie mag gegenwärtig noch in den Kinderschuhen stecken, lässt aber aus Sicht einiger Autoren für die Zukunft einige Fortschritte erwarten. ${ }^{30} \mathrm{Ihr}$ Ziel besteht im Wesentlichen darin, eine - wie der Name schon sagt - „Dämpfung“ des gefühlten Leides zu ermöglichen, um so den negativen Einfluss der Enhancement-Technologien auf das Wohlergehen und damit auf den Sinn auf ein Minimum reduzieren.

Und die moralische Schuld? Nagel nennt diese Implikation des EnhancementProjekts als dritte Möglichkeit, wie die erweiterten Wahlmöglichkeiten zu Einbußen im Wohlergehen führen könnten. Der Grundgedanke ist der folgende: Wenn unsere Wahlmöglichkeiten steigen, ergibt sich daraus auch eine größere Möglichkeit, dass wir moralische Entscheidungen treffen müssen, für die wir uns nachher ggf. schuldig fühlen. Denken wir etwa an Hilfeleistungen in einer Notsituation (z. B. einen Verkehrsunfall), in der jemand effizienter, aufmerksamer und gedankenschneller gehandelt hätte, wenn er zum Beispiel pharmakologische „Enhancer“ eingenommen hätte. Das ist eine ernstzunehmende Herausforderung, da gerade Gefühle wie moralische Schuld und das Versagen in einer moralischen Entscheidungssituation unser

\footnotetext{
28 Vgl. für eine Liste von mildernden Faktoren, die einer empirischen Überprüfung standhalten, Scheibehenne et al. (2010, S. 419-421).

29 Es ist klar, dass jemand, der dem Enhancement-Projekt insgesamt skeptisch gegenübersteht, einige Reserven haben dürfte, das Enhancement-Projekt zu verteidigen, indem weiteres Enhancement (z. B. durch memory dampening) betrieben wird. In diesem Zusammenhang möchte ich darauf hinweisen, dass ich in diesem Beitrag von einem neutralen Standpunkt ausgehe. Ich schlage mich weder auf die Seite der allgemeinen Befürworter noch auf die Seite der Kritiker, sondern versuche die Argumentationslage mit Bezug auf die Sinndebatte ohne Rückgriff auf eine Position in der Gesamtdebatte, die zudem substanzielle Argumente jenseits dieses Beitrages voraussetzen würde, darzustellen.
}

30 Vgl. zu dieser Technik und ihren Erfolgsaussichten Danaher (2014, S. 234). 
Wohlergehen in einer nicht unbeträchtlichen Weise beeinflussen dürften. Gleichwohl stehen wir diesem Phänomen nicht hilflos gegenüber. Uns stehen vielmehr Wege zur Verfügung, den Einfluss auf das Wohlergehen und damit auch die sinnminimierende Kraft abzusenken. Wie sollte das möglich sein? Eine Möglichkeit habe ich schon erläutert. Sie besteht darin, auf Techniken des memory dampening zurückzugreifen. Eine andere, die ich zusätzlich noch erwähnen möchte, könnte darin gesehen werden, auf eine bewährte Praxis zu setzen, die wir in vielen anderen Kontexten auch anwenden - nämlich die Distribution der Schuld. Die Grundidee besteht darin, die moralische Schuld nicht auf ein Individuum, sondern auf mehrere Köpfe zu verteilen. Im Fall einer fehlerhaften Hilfe in einer Notsituation wird etwa die rechtlich festzustellende Schuld in der Regel nicht ausschließlich beim Helfenden gesucht, sondern auch an anderen Faktoren festgemacht (z. B. den Umständen der Situation, dem Opfer, den anderen Helfenden, der möglichweise involvierten Technik, usw.). Ähnlich könnte auch im Rahmen des Enhancement-Projekts verfahren werden, wenngleich die Details der Distribution natürlich noch ausgearbeitet werden müssten. ${ }^{31}$ Im Kern könnte eine solche Praxis aber, wenn sie in das mindset des betreffenden Individuums einsickert, den emotionalen „Einschlag“ der Schuldgefühle erheblich senken und damit die Defizite im Wohlergehen und Sinn überschaubar machen.

\section{Mangelnder Einsatz und fehlende narrative Einheit}

Ein dritter und nunmehr letzter Versuch, die sinnminimierende Kraft des Enhancement-Projekts herauszuarbeiten, nimmt seinen Ausgangspunkt nicht bei den mentalen Zuständen des Subjekts, sondern bei den individuellen Charaktereinstellungen und der narrativen Struktur des eigenen Lebens. Hierbei handelt es sich offensichtlich um andere Aspekte des Wohlergehens, die sich vielleicht am ehesten - folgen wir Parfit - nicht im Rahmen des Hedonismus oder der Wunschtheorie, sondern der Objektive-Listen-Theorie analysieren lassen. ${ }^{32}$ Es gibt bestimmte Dinge in unserem Leben, die es sinnvoll machen (z. B. Charaktereinstellungen und die narrative Struktur des eigenen Lebens), und zwar unabhängig davon, ob sich diese gut anfühlen (Hedonismus) oder von uns erfolgreich gewünscht werden (Wunschtheorie). Beginnen wir mit den Charaktereigenschaften. Welche könnten sinnminimierend wirken und wie ist der Zusammenhang zu denken? Eine viel diskutierte Charaktereigenschaft ist etwa das Laster der Faulheit oder des mangelnden Engagements. Diese

\footnotetext{
31 Vgl. dafür etwa die ausführlichen Überlegungen in Vincent (2013), in denen zudem noch auf weitere Möglichkeiten eingegangen wird, den Einfluss von Schuldgefühlen für das Enhancement-Projekt zu minimieren.

32 Es gibt natürlich noch zahlreiche weitere sinnminimierende Faktoren im Rahmen einer Objektive-Listen-Theorien, die für den Sinn eine Rolle spielen könnten. Eine Möglichkeit bestünde etwa darin, nicht auf Charaktereigenschaften, sondern auf Fähigkeiten, insbesondere im Zusammenhang mit der eigenen Autonomie abzustellen. Siehe hierfür etwa Schermer (2008), Kipke (2011, Kap. 6.1, 6.4) und Leefmann (2017, bes. Kap. 4.2). Diese Diskussion bedarf sicherlich einer eigenen ausführlichen Berücksichtigung. Im Zusammenhang mit diesem Beitrag konzentriere ich mich jedoch auf die Charaktereigenschaften, insbesondere auf die Faulheit und des mangelnden Engagements, da diese bereits auf eine Literaturbasis im Kontext der Sinndebatte zurückgreifen können.
} 
Charaktermängel werden vielleicht am nachdrücklichsten von Susan Wolf in den Diskurs eingeführt. Das Beispiel, an dem sie die sinnminimierende Kraft festmacht, ist der sogenannte Blob. Darunter versteht sie ein Individuum, dessen Leben ,is lived in hazy passivity (...) at a not unpleasant level of consciousness (...) but unconnected to anyone or anything" (Wolf 2016a, S. 116). Nach Wolf kann man sich den Blob etwa als einen Couch-Potatoe vorstellen, der sein Leben vor dem Fernseher verbringt, Alkohol trinkt und Comedy-Sitcoms schaut. Ein Aspekt, warum Wolf ein solches Leben für sinnlos hält, besteht darin, dass der Blob schlicht faul und wenig engagiert ist. ${ }^{33}$ Es ist zu leicht und erfordert zu wenig Anstrengung, vor dem Fernseher zu sitzen und Bier zu trinken, als dass ein solches Leben noch als sinnvoll durchgehen kann. Wolf bezieht das Beispiel des sinnlosen Lebens freilich nicht auf das Enhancement-Projekt. Die Verbindung ist jedoch von anderen hergestellt worden. ${ }^{34}$ Der Grundgedanke ist hierbei, dass das Enhancement-Projekt dazu führt, dass wir uns im Fall seines Erfolges keiner Herausforderung mehr gegenübersehen, weil wir durch unseren optimierten Körper und Geist alles mit Leichtigkeit erreichen können. Ähnlich wie der Blob haben wir sprichwörtlich mit einem kleinen Fingerschnippen bereits alles, was uns wertvoll erscheint. Ein hoher Einsatz oder ein besonderes Engagement sind dafür nicht notwendig. Wir werden daher - so lässt sich das Argument abschließen - faul, träge und desengagiert und das sind Charaktereigenschaften, die die Sinnbilanz unseres Lebens erheblich nach unten ziehen. Ist das plausibel? Das kann aus mehreren Richtungen hinterfragt werden. Da wäre zum Beispiel die normative Rolle des Einsatzes und der eigenen Anstrengung. Handelt es sich hierbei um eine notwendige Bedingung für Sinn? Das kann man bezweifeln. ${ }^{35}$ Aber gewähren wir an dieser Stelle einen Ausgangskredit und nehmen an, dass der eigene Einsatz für den Sinn relevant ist. In diesem Fall lässt sich hinterfragen, ob es wahrscheinlich ist, dass das Enhancement-Projekt tatsächlich dazu führt, dass wir solch sinnminimierende Charaktereigenschaften entwickeln. Das scheint mir jedenfalls keineswegs ausgemacht zu sein. Ich folge in diesem Punkt John Danaher, der darauf hinweist, dass das Enhancement-Projekt keinen Freifahrtschein ins Land des Müßiggangs darstellt, da ,deciding to make use of enhancement technologies is itself going to be an effortful process, but also because it may open up newer, more effortful challenges for us to pursue" (Danaher 2014, S. 241). Es ist sicherlich nicht abzustreiten, dass uns einige Dinge leichter fallen werden. Darüber hinaus eröffnen sich jedoch auch neue Herausforderungen, in denen kognitiver Aufwand und ,harte Arbeit" weiterhin eine große Rolle spielen können.

Nun zur narrativen Struktur des eigenen Lebens: Diese wird von einigen als eine notwendige Bedingung des sinnvollen Lebens gedeutet. Prominent ist etwa die These von Thaddeus Metz, der versucht aufzuzeigen, dass es im Leben nicht nur darauf ankommt, solitäre sinnstiftende Handlungen auszuüben, sondern auch, dass diese

\footnotetext{
33 Einen weiteren Aspekt sieht Wolf darin, dass das Fernsehschauen des Blobs keine sinnstiftende Handlung darstellt. Vgl. für die hybride Sinntheorie von Wolf, in der sowohl subjektive als auch objektive Sinnquellen angenommen werden, klassisch Wolf (1997a, b) und die neueren Überlegungen in Wolf (2016b).

34 Vgl. Douglas (2013) und Danaher (2014, S. 240).

35 Vgl. für einen Überblick über die Kritik des Arguments Heinrichs et al. (2021, Abschnitt II, Kap. 5.1.2.6).
} 
Handlungen im Rahmen einer narrativen Struktur miteinander verbunden sind.$^{36}$ Was hat das mit dem Enhancement-Projekt zu tun? Eine Möglichkeit, die beiden Diskurse zu verbinden, besteht darin, das Enhancement-Projekt als Gefahr für die narrative Struktur und damit auch für den Sinn zu begreifen. Und das mag auch gar nicht so weit hergeholt sein, denn mit der Selbstverbesserung gehen auch Veränderungen unserer Anlagen, Einstellungen oder Erinnerungen einher. Das könnte es, so lässt sich das Argument rekonstruieren, schwermachen, das eigene Leben weiterhin als zusammenhängendes Ganzes zu begreifen.

Was ist davon zu halten? Zunächst einmal wäre zu klären, welche Art von $\mathrm{Zu}$ sammenhang eigentlich relevant ist. Die Diskussion um den Begriff der ,narrativen Einheit" im Kontext der Bioethik füllt mittlerweile Regalmeter. ${ }^{37}$ Man könnte an dieser Stelle an sehr lose Verbindungen zwischen den verschiedenen Lebensabschnitten denken, die lediglich dadurch gekennzeichnet sind, dass sich das Individuum darin „wiedererkennt“. Das wäre etwa der Fall, wenn das Leben des Individuums durch zahlreiche biographische Brüche gekennzeichnet ist. Solche Extremfälle scheinen für das Enhancement-Projekt jedoch wenig problematisch zu sein. Es ist jedenfalls nur schwer zu sehen, wie eine solch lose Struktur durch die Veränderungen unserer Anlagen, Fähigkeiten oder Erinnerungen herbeigeführt werden könnte. Anders sieht es freilich aus, wenn die geforderte narrative Struktur anspruchsvoller ausgelegt wird, etwa in der Weise, dass sie einen kohärenten Lebensplan beinhalten muss, in dem die Teile zueinander passen und möglicherweise aufeinander aufbauen. In diesem Fall ist die Herausforderung für das Enhancement-Projekt größer, allerdings auch nicht unlösbar. So hatte ich in einem der obigen Abschnitte schon darauf hingewiesen, dass eine Anpassung der Enhancement-Techniken durchaus gewinnbringend sein kann und viele Probleme möglicherweise zu lösen vermag, die sich stellen könnten. ${ }^{38}$ Nehmen wir wiederum den Fall des memory dampening: Hierbei handelt es sich um eine Technologie, die vermeintlich die narrative Einheit eines Lebens aufheben kann, da sie die Erinnerungsfähigkeit beeinträchtigt. Allerdings ist das nicht die einzige Möglichkeit. Sie kann etwa auch auf eine Weise angepasst werden, dass sie sich nicht auf das deklarative Erinnerungsvermögen bezieht, mit anderen Worten: Es wird lediglich die emotionale „Wucht“ der Erinnerung, aber nicht der kognitive Gehalt der Erinnerung selbst eliminiert. ${ }^{39}$ Die Erinnerung kann damit weiterhin Teil der narrativen Struktur bleiben. Das Enhancement-Projekt ist also auch gegenüber anspruchsvolleren Konzeptionen der narrativen Einheit keineswegs blind.

\footnotetext{
36 Vgl. Metz (2013a, Kap. 2).

37 Vgl. exemplarisch für die in diesem Zusammenhang relevanten Beiträge von Kraemer (2013), Nyholm und O'Neill (2016), Pugh et al. (2017).

38 Vgl. die Überlegungen zum Abschnitt „Entscheidungskonfusion, residuales Bedauern und moralische Schuld".

39 Vgl. dafür auch die ausführlichere Auseinandersetzung in Erler (2011).
} 


\section{Zusammenfassung, kritische Nachfragen und Ausblick}

Der Anspruch dieses Beitrages bestand darin zu prüfen, ob das Enhancement-Projekt einen negativen Einfluss auf das sinnvolle Leben haben könnte. Dafür wurde eine bestimmte Gruppe von Sinnträgern, die dem eigenen Wohlergehen zuzuordnen sind, herangezogen. Hierbei hat sich gezeigt, dass die geprüften Aspekte des Wohlergehens die Sinndimension eines Lebens nicht bedrohen, geschweige denn, ein Leben sinnlos machen können. Das liegt vor allem an zwei Gründen: Zum einen erscheint es unwahrscheinlich, dass die dafür notwendigen Effekte eintreten. Es scheint wenig plausibel zu sein, dass zum Beispiel das Enhancement-Projekt zu einer dauerhaften Unzufriedenheit mit sich selbst und der umgebenden Welt führt oder einen negativen Einfluss auf den eigenen Charakter hat; zum anderen kann die sinnminimierende Kraft, wenn sie tatsächlich auftreten sollte, durch eine Anpassung des Enhancement-Projekts abgemildert werden. Der Verfechter des EnhancementProjekts ist den Vorwürfen, dass möglicherweise Leidgefühle (z. B. Konfusion, Bedauern und Schuld) auftreten könnten oder die eigene Lebensführung inkohärent zu werden droht, nicht schutzlos ausgeliefert, sondern kann im Rahmen des eigenen Projekts darauf reagieren.

Nun werden nicht alle Kritiker ein solches Ergebnis klaglos hinnehmen. Ich antizipiere vor allem zwei ernstzunehmende Repliken: Die erste Replik geht von der Methode dieses Beitrages aus und könnte meine probabilistische Argumentationsweise infrage stellen. Kann diese wirklich zeigen, dass es unwahrscheinlich ist, dass die sinnminimierenden Effekte auftreten? Steht nicht im Wesentlichen das Wort der Kritiker gegen meines? Die Kritiker des Enhancement-Projekts halten es für wahrscheinlich, dass bestimmte sinnminimierende Faktoren auftreten; ich halte es für unwahrscheinlich. Nun glaube ich, dass ich nicht nur Vermutungen, sondern auch Gründe für diese These angeführt habe. Es steht mithin nicht die eine Psychologie aus dem Lehnstuhl einer anderen gegenüber. Es gibt vielmehr handfeste empirische Evidenzen dafür, dass die sinnminimierenden Faktoren aller Wahrscheinlichkeit nach nicht mit dem Enhancement-Projekt verbunden sind. Eine Replik, die meine Überlegungen lediglich mit dem Hinweis kontert, sie seien Gedankenspielereien, nimmt den Kern der Überlegungen nicht ernst. Es müssen auch empirische Belege für die gegenteilige Ansicht angeführt werden.

Eine zweite Replik könnte sich daran stoßen, dass ich bisher wenig dazu gesagt habe, wie mit dem Umstand umzugehen ist, dass das Enhancement-Projekt für einige Personen trotz aller Bemühungen mit Sinneinbußen verbunden sein könnte. Diese Möglichkeit ergab sich aus zwei Gründen: Zum einen gehe ich lediglich, wie mittlerweile deutlich geworden sein sollte, von Wahrscheinlichkeiten aus. Es gibt mithin keine $100 \%$ ige Sicherheit, auch dann nicht, wenn die Enhancement-Technologien selbst (z.B. das memory dampening) so modifiziert werden, dass sie die Wahrscheinlichkeit des Sinnverlusts auf ein Minimum reduzieren. Zum anderen scheint es auch, wie am Beispiel der Hauskeller-Diskussion gezeigt wurde, einen Personenkreis zu geben, für den das Risiko auf Sinnverlust höher ist, da sie jedwedem downscaling, also jeder möglichen Einschränkung der Enhancement-Technologien, kritisch gegenüberstehen. Das mag etwa für manche Trans- oder Posthumanisten der Fall sein. Wie ist, so könnte gefragt werden, mit diesem Befund umzugehen? 
Muss ich hierzu nicht auch eine These vertreten? Ich glaube nicht, dass das der Fall ist. Um das zu sehen, müssen wir uns daran erinnern, dass dieser Beitrag auf der Ebene der Individual- und nicht auf der Ebene der Sozialethik argumentiert. Das bedeutet, dass die Frage ausgeblendet wird, ob das Enhancement-Projekt zum Beispiel in irgendeiner Weise das - wie auch immer ausgestaltete - sinnvolle Zusammenleben der Bürgerinnen und Bürger oder ein - wie auch immer verstandenes sinntragendes Gemeinwesen bedroht. Es geht vielmehr um die Frage, ob die Technologien der Selbstverbesserung einen negativen Einfluss auf das sinnvolle Leben des Individuums haben. Aus dieser individualethischen Perspektive ist erst einmal nur zur Kenntnis zu nehmen, dass im intersubjektiven Vergleich einige Differenzen im möglichen Sinnverlust vorliegen können. Erst in einer sozialethischen Perspektive, die nicht Gegenstand dieses Beitrages ist, muss nachrangig die Frage beantwortet werden, ob das für das soziale Zusammenleben eine Bedrohung darstellt und wie möglicherweise regulatorisch darauf zu reagieren ist. Man kann diese Auslassung daher bedauern. Vor dem Hintergrund der methodischen Ausgangssituation ist sie jedoch nicht zu bemängeln.

Darüber hinaus sollen meine Klarstellungen aber nicht den Eindruck erwecken, als ob dieser Beitrag ein sich selbst tragendes System von notwendig aufeinander folgenden Überlegungen darstellt. Viele Thesen und Argumente geben noch Anlass für berechtigte Nachfragen, die in zukünftigen Arbeiten zu berücksichtigen sind. Hierzu gehören etwa die folgenden drei:

Erstens liegt es nahe, die Rolle des Wohlergehens für das sinnvolle Leben zu hinterfragen. Zur Erinnerung: Ich habe in diesem Beitrag vorausgesetzt, dass einige Aspekte des Wohlergehens eine bedeutende Rolle spielen können, wenn es darum geht, das sinnvolle Leben zu bestimmen. Genauer gesagt, bin ich stillschweigend davon ausgegangen, dass der Verlust mancher Aspekte des Wohlergehens die Sinnbilanz eines Lebens in nicht unerheblicher Weise herabsenkt. Die Prämisse dieser Argumentation sollte jedoch auch zum Gegenstand der Diskussion gemacht werden, insbesondere auch um das genaue normative „Gewicht“ des Wohlergehens für den Sinn einschätzen zu können.

Zweitens wäre zu prüfen, ob das Enhancement-Projekt tatsächlich nicht nur sinnminimierend, sondern auch sinnfördernd wirken kann. Ob man das für plausibel hält, mag sicherlich auch daran liegen, welche Sinntheorie man vertritt. Es scheint jedoch auch einiges dafür zu sprechen, dass der positive Einfluss des Enhancement-Projekts auf den Sinn zumindest bei den großen Theorieblöcken - wie der Deontologie und dem Konsequenzialismus - gegeben ist. ${ }^{40}$ Wenn sich das bestätigt, wäre ebenso darauf einzugehen, wie sich die „Sinnräuber“ im Verhältnis zu den Sinnquellen verhalten, also wie negative und positive Aspekte gegeneinander abgewogen werden können.

Drittens wurde der theoretische Blick auf die Sinnträger im Bereich des Wohlergehens eingeengt. Es wäre aber ebenso zu reflektieren, ob das Enhancement-Projekt nicht auch noch Einfluss auf weitere Dimensionen haben könnte. Zwar hängt auch

\footnotetext{
40 Vgl. für einen Versuch, den sinnstiftenden Charakter des Enhancement-Projekts herauszustellen und diesen gleichsam sowohl auf deontologische als auch konsequenzialistische Ausrichtungen zu beziehen, Danaher (2014, S. 239-241).
} 
das wiederum davon ab, welche Sinntheorie vertreten wird, also davon, welche Dimensionen für relevant gehalten werden. Häufig genannte Kandidaten, von denen zumindest die meisten ausgehen, dass sie irgendeinen Einfluss auf das sinnvolle Leben haben, sind etwa die Dimensionen der Moral oder des kosmischen Sinns, aber auch solche, die mit den individuellen Lebensleistungen (z. B. den eigenen Errungenschaften) oder den Arten der Handlungen (z. B. deren grundsätzlicher Ausrichtung auf einen werthaften Gegenstand) zusammenhängen. Diese Dimensionen oder Gesichtspunkte gilt es im Rahmen einer finalen Gesamtbewertung des EnhancementProjekts zu berücksichtigen.

Den Erkenntnisgewinn der hier vorliegenden Überlegungen sollten die genannten Desiderate jedoch nicht schmälern. Es wurde gezeigt, dass eine häufig beschrittene Argumentationslinie für den Kritiker des Enhancement-Projekts wenig Aussicht auf Erfolg hat. Es ist unwahrscheinlich, dass die technologische Selbstverbesserung dazu führt, dass unser Wohlergehen darunter leidet und unserem Leben dadurch der Sinn entzogen wird. Und in den Fällen, wo es doch zu befürchten ist, kann die Konsequenz durch einige Eingriffe in das Enhancement-Projekt selbst vermieden werden. Bedroht also - um die Eingangsfrage des Beitrages wiederaufzunehmen das Enhancement-Projekt das sinnvolle Leben? Diese Befürchtung kann, so das zentrale Ergebnis, für die sinnminimierende Kraft des Wohlergehens zurückgewiesen werden.

Funding Open Access funding enabled and organized by Projekt DEAL.

Open Access Dieser Artikel wird unter der Creative Commons Namensnennung 4.0 International Lizenz veröffentlicht, welche die Nutzung, Vervielfältigung, Bearbeitung, Verbreitung und Wiedergabe in jeglichem Medium und Format erlaubt, sofern Sie den/die ursprünglichen Autor(en) und die Quelle ordnungsgemäß nennen, einen Link zur Creative Commons Lizenz beifügen und angeben, ob Änderungen vorgenommen wurden.

Die in diesem Artikel enthaltenen Bilder und sonstiges Drittmaterial unterliegen ebenfalls der genannten Creative Commons Lizenz, sofern sich aus der Abbildungslegende nichts anderes ergibt. Sofern das betreffende Material nicht unter der genannten Creative Commons Lizenz steht und die betreffende Handlung nicht nach gesetzlichen Vorschriften erlaubt ist, ist für die oben aufgeführten Weiterverwendungen des Materials die Einwilligung des jeweiligen Rechteinhabers einzuholen.

Weitere Details zur Lizenz entnehmen Sie bitte der Lizenzinformation auf http://creativecommons.org/ licenses/by/4.0/deed.de.

\section{Einhaltung ethischer Richtlinien}

Interessenkonflikt M. Rüther gibt an, dass kein Interessenkonflikt besteht.

Ethische Standards Für diesen Beitrag wurden vom Autor keine Studien an Menschen oder Tieren durchgeführt. Für die aufgeführten Studien gelten die jeweils dort angegebenen ethischen Richtlinien.

\section{Literatur}

Buchanan A (2011) Beyond humanity? The ethics of biomedical science. Oxford University Press, Oxford Danaher J (2014) Hyperagency and the good life-Does extreme enhancement threaten meaning? Neuroethics 7(2):227-242. https://doi.org/10.1007/s12152-013-9200-1 
Douglas T (2013) Enhancing moral conformity and enhancing moral worth. Neuroethics 7(1):75-91. https://doi.org/10.1007/s12152-013-9183-y

Erler A (2011) Does memory modification threaten our authenticity? Neuroethics 4(3):235-249. https:// doi.org/10.1007/s12152-010-9090-4

Fletcher G (2015) Introduction. In: Fletcher G (Hrsg) The Routledge handbook of philosophy of wellbeing. Routledge, Abingdon, S 1-6

Hauskeller M (2011) Human enhancement and the giftedness of life. Philos Pap 40(1):55-79. https://doi. org/10.1080/05568641.2011.560027

Hauskeller M (2013) Better humans? Understanding the enhancement project. Acumen, London

Haybron D (2008) The pursuit of unhappiness. The elusive psychology of well-being. Oxford University Press, Oxford

Heinrichs JH, Rüther M, Stake M (2021) Sachstandsbericht „Neuroenhancement“. Alber, Freiburg

Juengst ET, Moseley D (2021) Human enhancement and meaning in life. In: Landau I (Hrsg) The Oxford companion on meaning in life. Oxford University Press, Oxford

Kamm F (2009) What is and what is not wrong with enhancement? In: Savulescu J, Bostrom N (Hrsg) Human enhancement. Oxford University Press, Oxford

Keller S (2009) Welfarism. Philos Compass 4(1):82-95

Kipke R (2011) Besser werden: Eine ethische Untersuchung zu Selbstformung und Neuro-Enhancement. mentis, Paderborn

Kraemer F (2013) Me, myself and my brain implant: Deep brain stimulation raises questions of personal authenticity and alienation. Neuroethics 6(3):483-497. https://doi.org/10.1007/s12152-011-9115-7

Leefmann J (2017) Zwischen Autonomie und Natürlichkeit. Der Begriff der Authentizität und die bioethische Debatte um Neuro-Enhancement. Mentis, Münster

Metz T (2002) Recent work on the meaning of life. Ethics 112:781-814. https://doi.org/10.1086/340462

Metz T (2007) New developments in the meaning of life. Philos Compass 2(2):196-217. https://doi.org/ 10.1111/j.1747-9991.2007.00061.x

Metz T (2013a) Meaning in life: an analytic study. Oxford University Press, Oxford

Metz T (2013b) The meaning of life. In: Zalta EN (Hrsg) Stanford encyclopedia of philosophy. The Metaphysics Research Lab, Stanford. http://plato.stanford.edu/entries/life-meaning/. Zugegriffen: 16. Aug. 2021

Moore A (2015) Well-being. In: Pritchard D (Hrsg) Oxford bibliographies in philosophy. Oxford bibliographies online datasets. https://www.oxfordbibliographies.com/view/document/obo-9780195396577/ obo-9780195396577-0284.xml. Zugegriffen: 16. Aug. 2021

Nagel S (2010) Too much of a good thing? Enhancement and the burden of self-determination. Neuroethics 3:109-119. https://doi.org/10.1007/s12152-010-9072-6

Nyholm S, O'Neill E (2016) Deep brain stimulation, continuity over time, and the true self. Camb Q Healthc Ethics 25(4):647-658. https://doi.org/10.1017/S0963180116000372

Parfit D (1984) Reasons and persons. Oxford University Press, Oxford

Pugh J, Maslen H, Savulescu J (2017) Deep brain stimulation, authenticity and value. Camb Q Healthc Ethics 26(4):640-657. https://doi.org/10.1017/S0963180117000147

Rice CM (2013) Defending the objective list theory of well-being. Ratio 26:196-211. https://doi.org/10. 1111/rati.12007

Rüther M (2021a) Meaning in life oder: Die Debatte um das sinnvolle Leben - Überblick über ein neues Forschungsthema in der analytischen Ethik. Teil 1: Grundlagen. Z Philos Forsch 1(75):115-155

Rüther M (2021b) Meaning in life oder: Die Debatte um das sinnvolle Leben - Überblick über ein neues Forschungsthema in der analytischen Ethik. Teil 2: normativ-inhaltliche Fragen. Z Philos Forsch 2(75):316-354

Rüther M, Muders S (2016) Sinn und Wohlergehen. Jahrb Wiss Ethik 20(1):73-112

Rüther M (i. V.) Was macht das Leben sinnvoll? Grundlinien einer normativ-inhaltlichen Theorie des sinnvollen Lebens. Unveröffentlichte Habilitationsschrift, Universität Bonn

Sandel M (2007) The case against perfection. Harvard University Press, Cambridge

Scheibehenne B, Greifeneder R, Todd PM (2010) Can there ever be too many options? A meta-analytic review of choice overload. J Consumer Res 37:409-425. https://doi.org/10.1086/651235

Schermer M (2008) Enhancements, easy shortcuts, and the richness of human activities. Bioethics 22(7):355-363. https://doi.org/10.1111/j.1467-8519.2008.00657.x

Schwartz B (2004) The paradox of choice: why more is less. Harper Collins, New York

Smuts A (2013) The good cause account of the meaning of life. South J Philos 51(4):536-562. https://doi. org/10.1111/sjp.12044 
Vincent NA (2013) Enhancing responsibility. In: Vincent NA (Hrsg) Neuroscience and legal responsibility. Oxford University Press, New York, S 305-334

Wolf S (1997a) Happiness and meaning: two aspects of the good life. Soc Philos Policy 14(1):207-225. https://doi.org/10.1017/s0265052500001734

Wolf S (1997b) Meaning and morality. Proc Aristot Soc 97(3):299-315. https://doi.org/10.1111/14679264.00018

Wolf S (2016a) The meaning of lives. In: Benatar D (Hrsg) Life, death, and meaning: key philosophical readings on the big questions, 3. Aufl. Rowman \& Littlefield, Lanham, S 113-132

Wolf S (2016b) Meaningfulness: A third dimension of the good life. Found Sci 21(2):253-269. https://doi. org/10.1007/s10699-014-9384-9 